\title{
Anesthesia-Related Neurally Mediated Syncope in the Perioperative Period: Two Case Reports
}

\author{
Jae Hang Shim, Hyung Joon Park, Chang Wook Lee, Seong Ho Park, Yoon Hyuk Hwang, Woo Jong Shin, Woo Jae Jeon \\ Department of Anesthesiology and Pain Medicine, Hanyang University College of Medicine, Seoul, Korea
}

\begin{abstract}
The following report describes the occurrence of severe hypotension and bradycardia in a healthy 50-year-old man following placement from supine to the beach chair position during general anesthesia for injured rotator cuff tendon repair, as well as the occurrence of micturition syncope in a 71-year-old man following postobstructive diuresis after combined spinal-epidural anesthesia for total knee replacement. Rapid decompression of the urinary bladder is associated with severe hypotension, bradycardia, and syncope. Neurally mediated syncope includes true vasovagal and situational syncope that can occur in association with various situations such as cough, micturition, postprandial state, and interscalene block. We report two cases of neurally mediated syncope (true vasovagal and situational) that occurred in different conditions and were controlled well with prompt and proper management without serious complications.
\end{abstract}

Keywords: Severe hypotension; Bradycardia; Syncope

\section{INTRODUCTION}

Syncope may have various origins, such as neurally mediated causes, postural hypotension, cardiac arrhythmias, organic heart disease, and cerebrovascular disease. Among these sources, neurally mediated syncope includes true vasovagal syncope and situational syncope, which can occur in association with a variety of situations such as blood loss, cough, sneeze, micturition, evacuation, postprandial state, and lifting a heavy load [1,2]. Micturition is clinically well-known as a factor that may cause hypotension, bradycardia, and if severe, syncope. The Bezold-Zarisch reflex (BJR) is a cardioinhibitory reflex that maintains tonic oppression in the vasomotor center and is characterized by three signs: vasorelaxation, bradycardia, and hypotension [3]. The combination of vasodilatation, bradycardia, and reflex cardiovascular depression is known by various terms including vasovagal syncope, BJR, and neurocardiogenic syncope [3].

In the present article, we report two cases of vasovagal syncope after obtaining written consent from the patients' family members. The first case report describes a healthy 50 -year-old man who underwent shoulder surgery under general anesthesia using sevoflurane and suddenly demonstrated severe bradycardia and hypotension after position change from supine to sitting after induction.

The other case is that of a 71-year-old male patient who underwent combined spinal-epidural anesthesia for total knee replacement. In this patient, the operation was performed without the awareness that an installed Foley catheter had been blocked. Severe bradycardia, hypotension, and loss of consciousness occurred immediately after the patient was transferred to the recovery room following micturition.

We report two cases of neurally mediated syncope (true vasovagal and situational) occurring under different conditions and controlled well with prompt and proper management without serious complications.

\section{CASE REPORTS}

\section{Case report 1}

The patient was male, aged 50 years, $169 \mathrm{~cm}$, and $72 \mathrm{~kg}$, and vis- 
ited the hospital to undergo shoulder surgery for a damaged rotator cuff. The patient had diabetes that had been diagnosed 10 years earlier, but preoperative laboratory findings were normal. Anesthesia was induced with pentothal sodium, fentanyl, and rocuronium bromide. For maintenance of anesthesia, $50 \% \mathrm{~N}_{2} \mathrm{O} / \mathrm{O}_{2}$, sevoflurane $3 \mathrm{vol} \%$, and remifentanil $0.05 \mu \mathrm{g} / \mathrm{kg} / \mathrm{min}$ were used. After stabilization of the vital signs, atropine sulfate $0.5 \mathrm{mg}$ was intravenously injected immediately prior to the positional change of the patient from supine to sitting position (beach chair position $\left.70^{\circ}-80^{\circ}\right)$. One or 2 minutes after placement of the patient in sitting position, heart rate suddenly decreased from 80 to 30 beats/min or less, with the blood pressure of 70/40 $\mathrm{mm} \mathrm{Hg}$. Intravenous injection of atropine sulfate $0.5 \mathrm{mg}$ was immediately performed, but heart rate and blood pressure did not change. After termination of the administration of $\mathrm{N}_{2} \mathrm{O}$ and sevoflurane, 1:10,000 epinephrine $1 \mathrm{~mL}(10 \mu \mathrm{g})$ was intravenously injected, and the patient was moved to the supine position. Heart rate soon recovered to 90 beats/min, and blood pressure returned to $120 / 60 \mathrm{~mm} \mathrm{Hg}$. Through discussion with the surgeons, the decision was made to postpone the operation. Detailed tests were performed, including 24-hour electrocardiography and echocardiography, and the patient was suspected of having vasovagal syncope. A $\beta$-blocker was administered for 1 week, and atropine sulfate and a stabilizer were administered as a preanesthetic. Following these steps, the operation was performed without complications.

\section{Case report 2}

A 71-year-old man was hospitalized to undergo total knee replacement owing to gonarthrosis. The patient was taking a benzodiazepine and a selective serotonin reuptake blocker because of an anxiety disorder, and he had a history of experiencing syncope a few times. Combined spinal-epidural anesthesia was performed. A mixture of $0.5 \%$ bupivacaine $(10 \mathrm{mg}$ ) and $0.1 \%$ epinephrine in $0.2 \mathrm{~mL}$ was injected intraspinal. The sensory block was verified up to the T4 neuromere. A forced-air warmer was used. However, the air warmer was removed after about 1 hour because the patient reported discomfort. For postoperative anesthesia, $8 \mathrm{~mL}$ of $0.15 \%$ levobupivacaine was injected through the epidural catheter. Although the total amount of fluid the patient received throughout the procedure was approximately $1,000 \mathrm{~mL}$, no urine output was observed during the 4 hours of surgery. Immediately prior to leaving the operating room, it was found that the tip of the Foley catheter was blocked on the patient side. When the Foley catheter was opened, about $500 \mathrm{~mL}$ of urine was discharged. At that time, vital signs were normal, and the patient's consciousness was clear. However, immediately after arrival in the recovery room, the patient's heart rate was drastically decreased from 70 to 30 beats/ min, blood pressure was 50/30 $\mathrm{mm} \mathrm{Hg}$, and consciousness was lost. Atropine sulfate $0.5 \mathrm{mg}$ and ephedrine $10 \mathrm{mg}$ were promptly administered, and the patient-controlled epidural analgesia was stopped. Following these measures, the patient's heart rate recovered to 60 beats/min and the blood pressure to $110 / 80 \mathrm{~mm} \mathrm{Hg}$, and subsequently, the consciousness was recovered.

\section{DISCUSSION}

BJR overlaps with vasovagal syncope, one type of neurocardiogenic syncope, which is caused by the reduction of venous return to the heart owing to decreased peripheral arterial pressure resulting from neurogenic mechanism [4]. Lower extremity muscle contraction is necessary for maintaining both venous return to the heart and blood pressure [3]. General anesthesia as well as epidural anesthesia inhibits such a "muscle pump" and the compensatory arterial and venous constriction. Venous congestion caused by the sitting position used during shoulder surgery under general anesthesia brings about decreased intraventricular volume, and a rapid decrease in preload causes abnormal myocardial contraction by the hypersecretion of catecholamine $[5,6]$. Enhanced myocardial contraction under reduced preload activates mechanical receptors of the heart, abnormally activates the parasympathetic nervous system, and induces repression of the sympathetic system. Subsequently, bradycardia occurs as a result of reflex vasorelaxation and vagus nerve activation. Paradoxical hypotension and bradycardia can be caused by intracardiac BJR activation because of blood flow dysfunction and vasodilation owing to venous blood stasis and the insufficiency of intracardiac blood that leads to vigorous cardiac contraction [4].

Severe cardiovascular collapse may be considered as a likely cause in the second case described in this report. However, based on the fact that the patient had no findings of cardiac abnormalities, the patient in this case might have experienced reflex micturition syncope. In our second case, considering the warm bed, the sensory block to the T4 neuromere, the hypovolemic state, the injection of levobupivacaine for postoperative analgesia, and the past history of syncope, it is assumed that micturition syncope caused the severe bradycardia, hypotension, and loss of consciousness 
that occurred upon the patient's arrival in the recovery room after micturition of about $500 \mathrm{~mL}$ of urine following the discovery of the blocked Foley.

Hainsworth [7] identified emotional stress, Valsalva maneuver, and reduced plasma volume as induction factors that caused syncope. In our second case, when the urinary bladder was full, abdominal pressure might have been increased, with all the intraperitoneal blood vessels affected by the increased pressure, and thus, the effect was similar to that of a Valsalva maneuver. In the blood vessels outside the abdominal cavity, the venous return might have been limited by the full urinary bladder, and cardiac output gradually reduced. Additionally, the warm blanket might have caused vasodilation, and thus, interrupted vasoconstriction. Moreover, it is assumed that opening the blocked Foley catheter caused bloodstream inhibition of the venous return by a loss in the stretch receptor stimulation. Furthermore, hypotension might have been exacerbated as the parasympathetic nerve was stimulated due to vasovagal activation. An insufficient supply of blood volume to the heart could cause vasoconstriction and tachycardia as part of the normal compensatory mechanism. However, paradoxical hypotension and bradycardia are induced by such mechanisms as bloodstream inhibition of the venous return and vasodilation from the stasis of the venous blood owing to the factors mentioned above, leading to the active contraction of the heart resulting from the BJR activation [8]. Kao and Racz [9] reported a case of loss of consciousness during micturition under general anesthesia. D' Alessio et al. [5] reported sudden bradycardia and hypotension caused by BJR owing to a reduction in venous return in the sitting position and increased cardiac contraction resulting from the use of epinephrine for the nerve block in a patient undergoing shoulder surgery in the sitting position under interscalene block. The diagnosis of vasovagal syncope is made using a head-up tilt table test. With the patient lying straight on the bed, blood pressure and electrocardiogram are monitored at 10-minute intervals for 5 minutes. The bed is then placed at a $70^{\circ}$ angle and blood pressure and electrocardiogram are monitored for 2 minutes every 30 minutes. Then, the patient is again laid down and isoproterenol is administered. The dose is gradually increased over 3 minutes for each dose from 1 to $5 \mu \mathrm{g}$ per minute. Blood pressure and electrocardiogram are observed at the time of dose increase and at $1 \mathrm{~min}$ ute and 3 minutes after each increase, and any abnormalities are then noted. As isoproterenol is administered, the patient's heart rate and blood pressure both gradually increase. In this process, the patient reports palpitations, nausea, vomiting, or chest discomfort. Usually, sinus tachycardia is observed. It is important to be aware that patterns such as atrial fibrillation or atrioventricular block may be shown on the electrocardiogram. The patient's bed should then be positioned again to $70^{\circ}$ while maintaining isoproterenol up to $5 \mu \mathrm{g}$. If the patient is positive for the test, blood pressure drops, and electrocardiogram changes such as bradycardia, cardiac arrest, and junctional rhythm occur. If the patient fails, the administration of isoproterenol should be terminated and the bed should be positioned upright again. Patients with negative reactions experience no subjective symptoms and show no changes in blood pressure and electrocardiogram. In this case, the blood pressure and electrocardiogram are monitored at 2-minute intervals for 10 minutes in bed posture while administering $5 \mu \mathrm{g}$ of isoproterenol. To prevent micturition syncope, exacerbating factors should be avoided to the greatest extent possible, and prodromal symptoms should be observed. Additionally, an elastic compression bandage can be applied to the lower extremities, an appropriate amount of fluid can be supplied, and a $\beta$-blocker can be taken, all of which may help. In addition, head-up tilt training and installation of permanent heartbeat instrumentation may helpful [10].

In conclusion, during the performance of anesthesia in a patient with a history of syncope who is scheduled to undergo lower extremity or shoulder surgery in a sitting position, a sufficient volume of fluid should be supplied, and factors that may reduce venous return should be eliminated. If necessary, in a patient in whom syncope is anticipated, it would be helpful to perform headup tilt testing using an inducing agent prior to the operation. Additionally, in the case of suspected syncope resulting from the intraoperative positional change or other causes, the patient should be quickly moved to the supine position, and the selection of appropriate drugs should be taken into consideration. Moreover, clinicians should be mindful of possible obstruction of the Foley catheter, when appropriate.

\section{REFERENCES}

1. Alboni P, Brignole M, Menozzi C, Raviele A, Del Rosso A, Dinelli M, et al. Clinical spectrum of neurally mediated reflex syncopes. Europace 2004; 6:55-62.

2. Brignole M, Alboni P, Benditt D, Bergfeldt L, Blanc JJ, Bloch Thomsen PE, et al. Guidelines on management (diagnosis and treatment) of syncope. Eur Heart J 2001;22:1256-306.

3. Kinsella SM, Tuckey JP. Perioperative bradycardia and asystole: relationship to vasovagal syncope and the Bezold-Jarisch reflex. Br J Anaesth 2001; 
86:859-68.

4. Campagna JA, Carter C. Clinical relevance of the Bezold-Jarisch reflex. Anesthesiology 2003;98:1250-60.

5. D'Alessio JG, Weller RS, Rosenblum M. Activation of the Bezold-Jarisch reflex in the sitting position for shoulder arthroscopy using interscalene block. Anesth Analg 1995;80:1158-62.

6. Ammirati F, Colivicchi F, Di Battista G, Garelli FF, Santini M. Electroencephalographic correlates of vasovagal syncope induced by head-up tilt testing. Stroke 1998;29:2347-51.
7. Hainsworth R. Pathophysiology of syncope. Clin Auton Res 2004;14 Suppl 1:18-24.

8. Abi-Samra F, Maloney JD, Fouad-Tarazi FM, Castle LW. The usefulness of head-up tilt testing and hemodynamic investigations in the workup of syncope of unknown origin. Pacing Clin Electrophysiol 1988;11:1202-14.

9. Kao YJ, Racz GB. Loss of consciousness after emergence from anaesthesia: a case of suspected micturition syncope. Anaesthesia 1990;45:738-40.

10. Parry SW, Kenny RA. The management of vasovagal syncope. QJM 1999; 92:697-705. 